\title{
Matrix nearness-based guaranteed passive system approximation
}

\author{
L. Knockaert ${ }^{1}$ \\ Dept. Information Technology, IBCN, Ghent University \\ Gaston Crommenlaan 8, PB 201, B-9050 Gent, Belgium
}

\begin{abstract}
In this paper we present a new approach towards global passive approximation in order to find a passive real-rational transfer function $G(s)$ that is an arbitrarily close approximation of the passive transfer function nearest to a non-passive square transfer function $H(s)$. It is based on existing solutions to pertinent matrix nearness problems. It is shown that the key point in constructing the passive real-rational transfer function $G(s)$, is to find a good rational approximation of the well-known ramp function over an interval defined by the minimum and maximum dissipation of $H(s)$. The proposed algorithms rely on the stable anti-stable decomposition of a given transfer function. Pertinent examples are given to show the scope and accuracy of the proposed algorithms.
\end{abstract}

Key words: Passivity, positive-real lemma, rational approximation

PACS: 84.30.-r, 84.32.-y

2010 MSC: 93D09, 41A20

\section{INTRODUCTION}

For linear time-invariant (LTI) systems, passivity guarantees stability and the possibility of synthesis of a transfer function by means of a lossy physical network of resistors, capacitors, inductors and transformers [1]. Therefore, passivity enforcement [2] and passivation (passification) [3] have become important issues in recent years [4-8], especially as more and more software tools render transfer functions which need passivity enforcement as a postprocessing step in order to generate reliable physical models. However, most of the techniques [2-7] are local perturbative and/or feedback approaches with fixed poles, while [8] is based on Fourier approximation, yielding passivated systems with a large number of poles.

In this paper we present a new global approach, in the sense that we obtain a passive real-rational transfer function $G(s)$ that is an arbitrarily close approximation of the passive transfer function nearest to the nonpassive transfer function $H(s)$. It is based on existing solutions to some pertinent matrix nearness problems $[9,10]$. We show that the key point in constructing the real-rational passive transfer function $G(s)$, is to find a good rational approximation for the ramp function $\max (0, x)$ over an interval defined by the minimum and maximum dissipation of the non-passive transfer function $H(s)$. It is also shown that in the Chebyshev or minimax sense this requires finding a rational Chebyshev approximation of the square root $\sqrt{x}$ over the interval $[0,1]$. The proposed algorithms rely heavily on the stable anti-stable decomposition $[11,12]$ of a given transfer function. A potential drawback of the present approach is that, in order to increase the accuracy of the passivation scheme, the number of poles of the passivated transfer function can be much higher than the number of poles of the original non-passive transfer function. Finally, six pertinent examples, both SISO and MIMO, are given to show the accuracy and relevance of the proposed algorithms.

\footnotetext{
Email address: luc.knockaert@intec.ugent.be (L. Knockaert)

${ }^{1}$ Corresponding author : tel. +3292643328, fax +3292649969 . This work was supported by a grant of the Research Foundation-Flanders (FWO-Vlaanderen)
} 


\section{PASSIVITY AND DISSIPATION}

In this Section we will discuss passivity of LTI systems and related passivity measures, such as minimum and maximum dissipation. The mathematical notation is as follows: throughout the paper $X^{T}$ and $X^{H}$ denote respectively the transpose and Hermitian transpose of a matrix $X$, and $I_{n}$ denotes the identity matrix of dimension $n$. The trace of a square matrix $X$, denoted $\operatorname{tr}(X)$ is the sum of its diagonal entries. A matrix is Hermitian if $X=X^{H}$ and unitary if $X X^{H}=X^{H} X=I$. For a Hermitian matrix $X$, we call $\lambda_{\min }(X)$ and $\lambda_{\max }(X)$ the minimum and maximum eigenvalue of $X$, respectively. The Frobenius norm is defined as $\|X\|_{F}=\sqrt{\operatorname{tr}\left(X^{H} X\right)}$ and the spectral norm (or 2-norm or maximum singular value) is defined as $\|X\|_{2}=\sqrt{\lambda_{\max }\left(X^{H} X\right)}$. It is easy to show that $\left\|X^{H}\right\|_{F}=\|X\|_{F}$ and $\left\|X^{H}\right\|_{2}=\|X\|_{2}$. For two Hermitian matrices $X$ and $Y$, the matrix inequalities $X>Y$ or $X \geq Y$ mean that $X-Y$ is respectively positive definite or positive semidefinite. The closed right halfplane $\Re e[s] \geq 0$ is denoted $\mathbb{C}_{+}$.

For the real system with minimal realization

$$
\begin{aligned}
& \dot{x}=A x+B u \\
& y=C x+D u
\end{aligned}
$$

where $B \neq 0, C \neq 0, D$ are respectively $n \times p, p \times n$ and $p \times p$ real matrices and $A \neq 0$ is a $n \times n$ real matrix, to be passive, it is required that the $p \times p$ transfer function

$$
H(s)=C\left(s I_{n}-A\right)^{-1} B+D
$$

is analytic in $\mathbb{C}_{+}$, such that

$$
H(i \omega)+H(i \omega)^{H} \geq 0 \quad \forall \omega \in \mathbb{R} .
$$

It is well-known [13] that the positive-real lemma in linear matrix inequalty (LMI) format : $\exists P^{T}=P>0$ such that

$$
\left[\begin{array}{cc}
A^{T} P+P A & P B-C^{T} \\
B^{T} P-C & -D-D^{T}
\end{array}\right] \leq 0
$$

guarantees the passivity of system (1). A necessary, but not sufficient, condition for passivity is that $A$ is stable, i.e., its eigenvalues are located in the closed left halfplane. In the sequel we will always suppose that $A$ is Hurwitz stable, i.e., its eigenvalues are located in the open left halfplane. We will also assume, unless otherwise stated, that $H(s)$ is non-passive, and devise ways of finding a passive transfer function $G(s)$ that is an arbitrarily close approximation of the passive transfer function nearest to $H(s)$.

In order to measure how far a given system is from being passive, we define the minimum dissipation $\delta_{-}(H)$ [14] as

$$
\delta_{-}(H)=\min _{\omega \in \mathbb{R}} \lambda_{\min }[R(\omega)]
$$

where

$$
R(\omega)=H(i \omega)+H(i \omega)^{H} .
$$

Similarly, we also define the maximum dissipation $\delta_{+}(H)$ as

$$
\delta_{+}(H)=\max _{\omega \in \mathbb{R}} \lambda_{\max }[R(\omega)] .
$$

It is clear that the system is passive if and only if $\delta_{-}(H) \geq 0$. If $\delta_{-}(H)<0$ the system is non-passive, and if $\delta_{+}(H) \leq 0$, the system is anti-passive, in the sense that then the system with transfer function $-H(s)$ is passive. When $\delta_{+}(H) \leq 0$, the nearest passive transfer function is simply $G(s)=0$.

In the sequel we will assume, unless otherwise stated, that the system is non-passive but not anti-passive, i.e., $-\infty<\delta_{-}(H)<0<\delta_{+}(H)<\infty$. To obtain $\delta_{-}(H)$ (or similarly $\delta_{+}(H)$ ), a simple bisection algorithm, based on the existence (or non-existence) of imaginary eigenvalues of the one-parameter Hamiltonian matrix

$$
\mathrm{N}_{\delta}=\left[\begin{array}{cc}
A & 0 \\
0 & -A^{T}
\end{array}\right]+\left[\begin{array}{c}
B \\
-C^{T}
\end{array}\right]\left(\delta I_{p}-D-D^{T}\right)^{-1}\left[\begin{array}{ll}
C & B^{T}
\end{array}\right]
$$

was proposed in [14]. We have 
Proposition 2.1. $\delta \geq \delta_{-}(H)$ if and only if $\mathrm{N}_{\delta}$ admits purely imaginary eigenvalues.

Proof. See [14].

It is clear that Proposition 2.1 always allows to decide, by checking the eigenvalues of $\mathrm{N}_{\delta}$, whether $\delta \geq \delta_{-}(H)$ or not. This forms the basis of the bisection algorithm of [14]. The only problem is to start with a so-called bracket, i.e., provable lower and upper bounds for $\delta_{-}(H)$. For this purpose we have

\section{Proposition 2.2.}

$$
-2\|H\|_{\infty} \leq \delta_{-}(H) \leq \lambda_{\min }\left(D+D^{T}\right) \leq \lambda_{\max }\left(D+D^{T}\right) \leq \delta_{+}(H) \leq 2\|H\|_{\infty} .
$$

The proof is straightforward. Here the infinity norm $\|H\|_{\infty}$ is defined as

$$
\|H\|_{\infty}=\max _{\omega \in \mathbb{R}}\|H(i \omega)\|_{2} .
$$

Note that we can replace $\|H\|_{\infty}$ in (2) by an upper bound such as the one given in [14], i.e.,

$$
\gamma_{\mathrm{ub}}=\|D\|_{2}+2 \sqrt{n \operatorname{tr}\left(W_{c} W_{o}\right)}
$$

where $W_{c}, W_{o}$ are the controllability and observability Grammians.

\section{MATRIX NEARNESS CONSIDERATIONS}

A matrix nearness problem consists of finding, for an arbitrary matrix, a nearest member of some given class of matrices, where distance is measured in a matrix norm [9]. The matrix nearness problem we consider here is finding the positive semidefinite Hermitian matrix nearest to a given Hermitian matrix. We have :

Theorem 3.1. Let $A=A^{H}$ be any Hermitian matrix with eigendecomposition $A=U \Lambda U^{H}$, with $U$ a unitary and $\Lambda$ a real diagonal matrix. Then the positive semidefinite Hermitian matrix nearest to $A$, both with respect to the Frobenius and spectral norms, is given by $A_{+}=U \max (0, \Lambda) U^{H}$.

Proof. First we give the proof for the Frobenius norm. We need to find

$$
\min _{X \geq 0}\|X-A\|_{F}
$$

Putting $X=U Y U^{H}$, and exploiting the unitary invariance of the Frobenius norm, we obtain

$$
\|X-A\|_{F}^{2}=\|Y-\Lambda\|_{F}^{2}=\sum_{i \neq j}\left|Y_{i j}\right|^{2}+\sum_{i}\left|Y_{i i}-\Lambda_{i i}\right|^{2} .
$$

It is clear that the minimum occurs when $Y_{i j}=0$ for $i \neq j$, in other words when $Y$ is diagonal. Hence we obtain

$$
\|X-A\|_{F}^{2}=\|Y-\Lambda\|_{F}^{2}=\sum_{i}\left|Y_{i i}-\Lambda_{i i}\right|^{2} .
$$

It is easy to see that we must take $Y_{i i}=\max \left(0, \Lambda_{i i}\right)$ and this completes the proof for the Frobenius norm. Note that

$$
\min _{X \geq 0}\|X-A\|_{F}=\sqrt{\sum_{\lambda_{i}(A)<0} \lambda_{i}(A)^{2}} .
$$

For the spectral norm, it is known $[9,10]$ that

$$
\min _{X \geq 0}\|X-A\|_{2}=\inf \{r \geq 0: A+r I \geq 0\} .
$$


In other words,

$$
\min _{X \geq 0}\|X-A\|_{2}=\max \left(0,-\lambda_{\min }(A)\right) .
$$

Now

$$
\left\|A_{+}-A\right\|_{2}=\max _{\lambda_{i}(A)<0}\left|\lambda_{i}(A)\right|
$$

which is zero when there are no negative eigenvalues, and $-\lambda_{\min }(A)$ when there are negative eigenvalues.

Remark 3.1. From Theorem 3.1 it is possible to find the point-wise nearest positive semidefinite matrix for the Hermitian matrix $R(\omega)=H(i \omega)+H(i \omega)^{H}$. Obviously, if we decompose $R(\omega)$ as

$$
R(\omega)=U(\omega) \Lambda(\omega) U(\omega)^{H}
$$

then the point-wise nearest positive semidefinite matrix is

$$
R_{+}(\omega)=U(\omega) \max (\Lambda(\omega), 0) U(\omega)^{H} .
$$

Unfortunately, in general, the entries of $R_{+}(\omega)$ will not consist of rational functions and therefore cannot represent the transfer function of an LTI model on the imaginary axis. This problem, which in fact amounts to a rational approximation problem, will be addressed in the next Section.

\section{RATIONAL APPROXIMATIONS}

Theorem 4.1. Let $H(s)$ be passifiable, i.e., $-\infty<\delta_{-}(H)<0<\delta_{+}(H)<\infty$, and let $R(\omega)=H(i \omega)+$ $H(i \omega)^{H}$. Let further $f(x)$ be a real-rational function ${ }^{2}$ satisfying

$$
0 \leq f(x)-\max (x, 0) \leq \alpha \quad \forall x \in\left[\delta_{-}(H), \delta_{+}(H)\right]
$$

for some finite positive $\alpha$. Then $f(R(\omega))$ is positive semidefinite for all $\omega \in \mathbb{R}$. Furthermore we have

$$
\left\|f(R(\omega))-R_{+}(\omega)\right\|_{2} \leq \alpha \quad \forall \omega \in \mathbb{R} .
$$

Proof. We have

$$
f(R(\omega))-R_{+}(\omega)=U(\omega)\{f(\Lambda(\omega))-\max (\Lambda(\omega), 0)\} U(\omega)^{H} \geq 0 .
$$

Since $R_{+}(\omega)$ is positive semidefinite, the same holds for $f(R(\omega))$. Next, since the spectral norm is unitarily invariant, we have

$$
\begin{aligned}
\left\|f(R(\omega))-R_{+}(\omega)\right\|_{2} & \leq \max _{i}\left|f\left(\lambda_{i}(\omega)\right)-\max \left(\lambda_{i}(\omega), 0\right)\right| \\
& \leq \max _{\omega \in \mathbb{R}} \max _{i}\left|f\left(\lambda_{i}(\omega)\right)-\max \left(\lambda_{i}(\omega), 0\right)\right| \\
& \leq \max _{x \in\left[\delta_{-}(H), \delta_{+}(H)\right]}\{f(x)-\max (x, 0)\} \leq \alpha
\end{aligned}
$$

where the last inequality follows from the fact that all $\lambda_{i}(\omega)$ are inside the interval $\left[\delta_{-}(H), \delta_{+}(H)\right]$. This completes the proof.

Theorem 4.1 shows that the matrix $R_{+}(\omega)$ can be approximated from above by the matrix $f(R(\omega))$. The problem is to find a suitable real-rational function $f(x)$. A potentially good candidate function $f(x)$ follows from:

\footnotetext{
${ }^{2} \mathrm{~A}$ real-rational function $f(x)$ is a rational function assuming only real values for all real $x$.
} 
Theorem 4.2. Let $\zeta_{n}(x)=x(1+x)^{n} /\left((1+x)^{n}-1\right)$. Then

$$
0 \leq \zeta_{n}(x)-\max (x, 0) \leq \frac{1}{n} \quad \forall x \geq-1, \quad n=1,2,3, \ldots
$$

Proof. First we prove that $\zeta_{n}(x)-x \geq 0$ for $x \geq 0$. We have

$$
\zeta_{n}(x)-x=\left(\frac{(1+x)^{n}-1}{x}\right)^{-1}
$$

which is a positive and decreasing function for $x \geq 0$. Next we show that $\zeta_{n}(x)$ is increasing for all $x \geq-1$. This is equivalent to proving that $\zeta_{n}(t-1)=\left(t^{n+1}-t^{n}\right) /\left(t^{n}-1\right)$ is increasing for all $t \geq 0$. This is clearly the case for $n=1$. Taking derivatives, we have

$$
\frac{d}{d t} \zeta_{n}(t-1)=\left[n-(n+1) t+t^{n+1}\right] \frac{t^{n-1}}{\left(t^{n}-1\right)^{2}}
$$

Now $n-(n+1) t+t^{n+1}$ equals $n$ when $t=0$ and $\infty$ when $t=\infty$. Since the derivative of $n-(n+1) t+t^{n+1}$ is $(n+1)\left(t^{n}-1\right)$, the function $n-(n+1) t+t^{n+1}$ attains its unique minimum (with value zero) at $t=1$. Hence $\zeta_{n}(x)$ is increasing for all $x \geq-1$. We therefore conclude that $\zeta_{n}(x)-\max (x, 0)$ increases from 0 to $1 / n$ in the interval $[-1,0]$, and decreases from $1 / n$ to 0 in the interval $[0, \infty]$, which completes the proof.

Corollary 4.1. Let $H(s)$ be passifiable. Then the real-rational function $f(x)=\nu \zeta_{n}(x / \nu)$ with $\nu=\left|\delta_{-}(H)\right|$ satisfies the premises of Theorem 4.1 with $\alpha=\nu / n$.

Proof. Straightforward.

Also, we need to define the matrix $f(R(\omega))=f\left(H(i \omega)+H(i \omega)^{H}\right)$ in the whole $s$-plane and then to extract a Hurwitz stable transfer function from it. By analytical continuation, we find the transfer function $V(s)=f\left(H(s)+H(-s)^{T}\right)$ in the entire $s$-plane. Since $f(x)$ is real-rational, the transfer function $V(s)$ generally represents a realizable ${ }^{3}$ per-symmetric LTI model, i.e., with transfer function satisfying $V(s)=V(-s)^{T}$. This implies that the poles of $V(s)$ admit the imaginary axis as symmetry axis. The following proposition shows how, starting from a per-symmetric LTI model $V(s)$ we can find a Hurwitz stable transfer function by additive decomposition $[11,12]$.

Proposition 4.1. Let $V(s)$ be per-symmetric, i.e., $V(s)=V(-s)^{T}$, such that $V(s)$ has no poles on the imaginary axis. Then $V(s)$ can be decomposed as $V(s)=X(s)+X(-s)^{T}$, where $X(s)$ is Hurwitz stable.

Proof. The proof is constructive, since we actually show how to obtain a Hurwitz stable transfer function $X(s)$ satisfying the premises. Putting $V(s)=V_{0}(s)+D_{0}$, where $V_{0}(s)$ is strictly proper and $D_{0}=D_{0}^{T}=$ $V(\infty)$, we can decompose $V_{0}(s)$ uniquely into its stable and anti-stable parts, i.e.,

$$
V_{0}(s)=X_{s t a b}(s)+X_{a n t i}(s) .
$$

Since $V_{0}(s)$ is per-symmetric we have

$$
X_{s t a b}(s)+X_{a n t i}(s)=X_{s t a b}(-s)^{T}+X_{a n t i}(-s)^{T}
$$

and hence, using the uniqueness of the decomposition, $X_{a n t i}(s)=X_{s t a b}(-s)^{T}$. It follows that $V(s)$ can be decomposed as $V(s)=X(s)+X(-s)^{T}$, with $X(s)=X_{\text {stab }}(s)+\frac{1}{2} D_{0}+E$, where $E$ is an arbitrary skew-symmetric matrix. It should be noted that the procedure is unique when the skew-symmetric matrix $E$ is known a priori.

\footnotetext{
${ }^{3}$ In fact, given any two real coprime polynomials $p(x), q(x)$, and a realizable transfer function $Z(s)$, the transfer functions $p(Z(s))$ and $q(Z(s))$ are realizable, and the same can be said for $f(Z(s))$ where $f(x)=p(x) / q(x)$, with the proviso that $q(Z(\infty))$ is nonsingular.
} 
Note that in the sequel we will always use the stable, anti-stable decomposition algorithm of [12].

Remark 4.1. Proposition 4.1 assumes that $V(s)$, in our case $V(s)=f\left(H(s)+H(-s)^{T}\right)$, does not admit poles on the imaginary axis. By the inequality constraints (3) we know that

$$
0 \leq f\left(\lambda_{i}(\omega)\right)-\max \left(\lambda_{i}(\omega), 0\right) \leq \alpha
$$

for all eigenvalues $\lambda_{i}(\omega)$ of $R(\omega)$. Since $H(s)$ is assumed Hurwitz stable, $R(\omega)=H(i \omega)+H(i \omega)^{H}$ cannot admit real poles, and hence, by the inequalities (4), the functions $f\left(\lambda_{i}(\omega)\right)$ are bounded. It follows that all entries of $V(i \omega)=f(R(\omega))$ are bounded, which implies that $V(s)$ cannot have poles on the imaginary axis.

\section{TWO PASSIVATION ALGORITHMS}

The following two algorithms, in contradistinction with the minimax algorithm of Section 6 , only use the minimum dissipation $\delta_{-}(H)<0$. By Theorem 4.1 and Corollary 4.1 we need to find an LTI model with transfer function $\phi_{n}\left(H(s)+H(-s)^{T}\right)$ where the real-rational function $\phi_{n}(x)$ of denominator degree $n$ and numerator degree $n+1$ is given by

$$
\phi_{n}(x)=\nu \zeta_{n}(x / \nu)=\frac{x(1+x / \nu)^{n}}{(1+x / \nu)^{n}-1}
$$

where $\nu=\left|\delta_{-}(H)\right|$. It is easy to show that the following recurrence relation holds :

$$
\phi_{2 n}(x)=\frac{\phi_{n}(x)^{2}}{2 \phi_{n}(x)-x} \quad n=1,2,3, \ldots
$$

with $\phi_{1}(x)=x+\nu$.

A first algorithm ( Algorithm 1) that comes to mind with $Z(s)=H(s)+H(-s)^{T}$ is :

Initial value :

$$
Z_{0}(s)=Z(s)+\nu I_{p}
$$

Loop :

$$
\text { for } \quad k=1 \quad \text { to } \quad n_{1}: \quad Z_{k}(s)=Z_{k-1}(s)\left(2 Z_{k-1}(s)-Z(s)\right)^{-1} Z_{k-1}(s) .
$$

The associated upper bound $\alpha_{k}$ at each step $Z_{k}(s), k=0,1, \ldots, n_{1}$, is $\alpha_{k}=\nu / 2^{k}$, and all $Z_{k}(i \omega)$ are, by construction, positive semidefinite. Since the $Z_{k}(s)$ are all per-symmetric, we can use Proposition 4.1 to decompose all (or only the $n_{1}$ th one) $Z_{k}(s)$ in their stable and anti-stable parts as

$$
Z_{k}(s)=Z_{k}^{s t a b}(s)+Z_{k}^{s t a b}(-s)^{T} .
$$

As a last, but necessary step, we must add the skew-symmetric matrix $\frac{1}{2}\left(D-D^{T}\right)$, since this matrix gets deleted when forming the sum $Z(s)=H(s)+H(-s)^{T}$. In other words, the passive Hurwitz stable approximant $G_{k}(s)$ is

$$
G_{k}(s)=Z_{k}^{s t a b}(s)+\frac{1}{2}\left(D-D^{T}\right)
$$

As a very simple, yet illustrative example take $k=0$. Since $Z_{0}(s)=H(s)+H^{T}(-s)+\nu I_{p}$, we obtain easily that

$$
\begin{aligned}
G_{0}(s) & =Z_{0}^{\text {stab }}(s)+\frac{1}{2}\left(D-D^{T}\right) \\
& =(H(s)-D)+\frac{1}{2}\left(D+D^{T}+\nu I_{p}\right)+\frac{1}{2}\left(D-D^{T}\right) \\
& =H(s)+\frac{\nu}{2} I_{p}
\end{aligned}
$$

which is passive by construction. Algorithm 1 can be summarized in the following steps : 


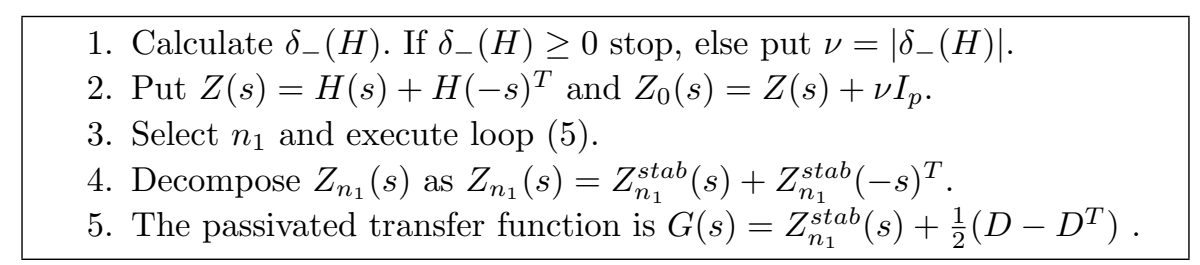

It should be noted that Algorithm 1, especially the loop (5), is carried out not on the transfer function level, but on the realization level, requiring two LTI model multiplications and one LTI model inversion (see [15] 3-13). Hence, in practice, Algorithm 1 has the important drawback that the realizations of the transfer functions $Z_{k}(s)$ in the algorithmic loop are not minimal in general — see Example 5.1.1 —, and hence it could happen that the stable anti-stable decomposition [12] might not perform well, due to the presence of artificially generated poles.

Before proposing a hopefully better algorithm, and in order to address the computational complexity of the passivated transfer function $G(s)$, we want to estimate the number of poles of $G(s)$. We suppose that $f(x)$ is an irreducible real-rational function with denominator degree $M$ and numerator degree $M+1$. In this paper this is always the case, see also Section 6. Hence, if we further suppose that all the poles are simple, we can decompose $f(x)$ into partial fractions as

$$
f(x)=\alpha_{0}+\beta_{0} x+\sum_{k=1}^{M} \frac{\alpha_{k}}{x-\beta_{k}} .
$$

Now if the original Hurwitz stable transfer function $H(s)$ has $N$ poles, then the transfer function $Z(s)=$ $H(s)+H(-s)^{T}$ has $2 N$ poles. Also, $f(Z(s))$ can be written as

$$
f(Z(s))=\alpha_{0} I_{p}+\beta_{0} Z(s)+\sum_{k=1}^{M} \alpha_{k}\left(Z(s)-\beta_{k} I_{p}\right)^{-1} .
$$

Hence, the set of poles of $f(Z(s))$ is at most the union of the sets of poles of $Z(s)$ and $\left(Z(s)-\beta_{k} I_{p}\right)^{-1}$. It is well known [15], that when a transfer function $H(s)$ is such that $H(\infty)$ is invertible, then $H(s)^{-1}$ exists and has the same number of poles as $H(s)$. Therefore, the number of poles of $f(Z(s))$, not considering potential pole-zero cancellations, is $2 N(M+1)$. Finally, after the stable anti-stable decomposition, this number is to be divided by two, yielding

$$
N_{G}=N(M+1)
$$

poles for the final passivated transfer function $G(s)$. Of course the number $N(M+1)$ is only an upper bound, since pole-zero cancellations can occur. If for some reason, the number of poles of the passivated transfer function $G(s)$ appears to be unacceptable high, a final judiciously chosen passivity preserving model order reduction step [16-20] could be applied.

Hence, in order to find a workable algorithm, we have to find the partial fraction decomposition of $f(x)=$ $\phi_{n}(x)=\nu \zeta_{n}(x / \nu)$. If we restrict ourselves to even $n=2 m \geq 2$, we have the partial fraction expansion

$$
\zeta_{2 m}(x)=x+\frac{1}{m}\left(\frac{1}{x+2}+\Re e \sum_{k=1}^{m-1} \frac{e^{2 \pi i k / m}-e^{\pi i k / m}}{x+1-e^{\pi i k / m}}\right) .
$$

This follows from the fact that the poles of $\zeta_{2 m}(z)$ are given by $z=e^{\pi i k / m}-1, k=1, \ldots, 2 m-1$. Hence, since $f(x)=\nu \zeta_{2 m}(x / \nu)$ we have

$$
f(Z(s))=Z(s)+\frac{\nu^{2}}{m}\left(\left[Z(s)+2 \nu I_{p}\right]^{-1}+\Re e \sum_{k=1}^{m-1}\left(e^{2 \pi i k / m}-e^{\pi i k / m}\right)\left[Z(s)+\nu\left(1-e^{\pi i k / m}\right) I_{p}\right]^{-1}\right) .
$$

Algorithm 2 performs the state space addition (7) as is, i.e., we add the realizations of $Z(s),\left(\nu^{2} / m\right)[Z(s)+$ $\left.2 \nu I_{p}\right]^{-1}$, etc., to obtain $f(Z(s))$. The explicit state space form for the terms

$$
\Re e\left[\left(e^{2 \pi i k / m}-e^{\pi i k / m}\right)\left[Z(s)+\nu\left(1-e^{\pi i k / m}\right) I_{p}\right]^{-1}\right]
$$


in formula (7) is obtained by the state space technique described in the Appendix. Finally, the stable antistable decomposition yields the passivated transfer function $G(s)$. Note that for Algorithm 2 the number of poles of the passivated transfer function is $N_{G}=2 \mathrm{Nm}$. Algorithm 2 can be summarized in the following steps :

1. Calculate $\delta_{-}(H)$. If $\delta_{-}(H) \geq 0$ stop, else put $\nu=\left|\delta_{-}(H)\right|$.

2. Put $Z(s)=H(s)+H(-s)^{T}$ and select $m \geq 1$.

3. Calculate $f(Z(s))$ by means of the state space addition $(7)$.

4. Decompose $f(Z(s))$ as $f(Z(s))=Y_{1}^{s t a b}(s)+Y_{1}^{s t a b}(-s)^{T}$.

5. The passivated transfer function is $G(s)=Y_{1}^{s t a b}(s)+\frac{1}{2}\left(D-D^{T}\right)$.

\subsection{Numerical Examples}

We will consider only reciprocal non-passive systems, i.e., systems with $H(s)=H(s)^{T}$, as these systems are representative of LTI systems satisfying the electromagnetic condition known as Lorentz reciprocity [21]. Of course the theory also remains valid for non-reciprocal LTI systems. Since for reciprocal systems $R(\omega)$ is real and even, this explains why the plots in the sequel only show values for non-negative frequencies.

\subsubsection{First example}

As a first example we take the SISO Hurwitz stable non-passive transfer function

$$
H(s)=\frac{s^{5}+7.2 s^{4}+47.01 s^{3}+230.8 s^{2}+536.6 s+587.1}{s^{5}+3.2 s^{4}+32.61 s^{3}+43.63 s^{2}+117.5 s+104.3}
$$

We use the approach of Algorithm 1 with $n_{1}=2$. The passivated approximation $G(s)$ has a non-minimal realization with 65 poles $^{4}$ which is reduced to 20 by pole-zero cancellation [15]. This means that Algorithm 1 artificially introduces 45 poles and 45 zeros which afterwards cancel out. The real and imaginary parts of the original transfer function $H(s)$ vs. the passivated transfer function $G(s)$ are shown in Figures 1 and 2. For comparison purposes, we also added the plots resulting from the passivity compensation algorithm of $[7]$.

\subsubsection{Second example}

As a second example we take the SISO Hurwitz stable minimum phase non-passive transfer function

$$
H(s)=\frac{(s+1)(s+3)(s+90)(s+95)(s+100)}{(s+25)(s+35)(s+38)(s+180)(s+185)} .
$$

We use the approach of Algorithm 2 with $m=5$. The passivated approximation $G(s)$ has a realization with $50=2 \times m \times 5$ poles. The real and imaginary parts of the original transfer function $H(s)$ vs. the passivated transfer function $G(s)$ are shown in Figures 3 and 4. For comparison purposes, we also added the plots resulting from the passivity compensation algorithm of [7].

\subsubsection{Third example}

As a third example we take the $2 \times 2$ MIMO Hurwitz stable non-passive transfer function

$$
H(s)=\left[\begin{array}{cc}
2+\frac{12}{s^{2}+3 s+2} & -\frac{2 s+10}{s+6} \\
-\frac{2 s+10}{s+6} & 2-\frac{s+3}{s^{2}+3 s+2}
\end{array}\right] .
$$

\footnotetext{
${ }^{4}$ We found tentatively by simulating with different values of $n_{1}$ that the number of poles generated by Algorithm 1 is given by the heuristic formula $5 \times \frac{3^{n_{1}+1}-1}{2}$. After pole-zero cancellation the number of poles reduces to $5 \times 2^{n_{1}}$.
} 
We use the approach of Algorithm 2 with $m=4$. The passivated approximation $G(s)$ has a realization with $48=2 \times m \times 6$ poles (here the number 6 is the McMillan degree of the realization of $H(s)$ ). Fig. 5 plots the values of $\lambda_{\min }\left(G(i \omega)+G(i \omega)^{H}\right)$ vs. $\lambda_{\min }\left(H(i \omega)+H(i \omega)^{H}\right)$. To show the nearness of the original and passivated transfer functions $H(s)$ and $G(s)$, we plot the relative error $\|G(i \omega)-H(i \omega)\|_{2} /\|H(i \omega)\|_{2}$ in Fig. 6 . For comparison purposes, we also added the plots resulting from the passivity compensation algorithm of $[7]$.

\section{MINIMAX PASSIVATION ALGORITHM}

The following minimax algorithm, unlike Algorithms 1 and 2 of Section 5, uses both the minimum dissipation $\delta_{-}(H)<0$ and the maximum dissipation $\delta_{+}(H)>0$. The starting point for obtaining a passive approximant is to find a real-rational function $f(x)$ that satisfies

$$
0 \leq f(x)-\max (x, 0) \leq \alpha \quad \forall x \in[-a, b]
$$

where $a=-\delta_{-}(H)=\left|\delta_{-}(H)\right|$ and $b=\delta_{+}(H)$. Since $\max (x, 0)=(|x|+x) / 2$, this can be written as

$$
-\alpha \leq 2 f(x)-x-\alpha-|x| \leq \alpha \quad \forall x \in[-a, b] .
$$

Putting $r(x)=2 f(x)-x-\alpha$, and since our aim is to find the smallest positive $\alpha$ such that (12) is satisfied, it is seen that we must find the rational minimax or Chebyshev approximant, i.e.,

$$
\min _{r} \max _{x \in[-a, b]}|r(x)-| x||
$$

Let us first treat the case $a=b=1$, which is well-documented in the literature [22-24]. Since $|x|$ is even and the interval $[-1,1]$ is symmetric with respect to 0 , it is clear that $r(x)$ must be an even rational function, i.e., $r(x)=\rho\left(x^{2}\right)$. If we take $\rho(t)=\rho_{n}(t)$ irreducible with numerator and denominator of exact degree $n$, the minimax problem can be reformulated as:

$$
\min _{\rho_{n}} \max _{0 \leq t \leq 1}\left|\rho_{n}(t)-\sqrt{t}\right|
$$

Calling $E_{n}$ the value obtained by the minimax problem (13), it is clear that at the minimum we must have

$$
-E_{n} \leq \rho_{n}(t)-\sqrt{t} \leq E_{n} \quad \text { for } \quad 0 \leq t \leq 1
$$

Furthermore, the Remes condition $[24,25]$ requires that there are exactly $2 n+2$ points $t_{k}$ inside $[0,1]$ where the equioscillation equality

$$
\sqrt{t_{k}}-\rho_{n}\left(t_{k}\right)=(-1)^{k} E_{n} \quad k=1,2, \ldots, 2 n+2
$$

is satisfied. This allows an iterative approach [24] to find the optimal $E_{n}$ and $\rho_{n}(t)$. The poles and zeros of $\rho_{n}(t)$ are all simple and intertwined on the negative real axis [26]. It follows that $\rho_{n}(t)$ can be generally written as

$$
\rho_{n}(t)=a_{0}-\sum_{k=1}^{n} \frac{a_{k}}{t+b_{k}}
$$

where all $a_{k}, b_{k}{ }^{5}$ are positive. For $n=4$ the coefficients $a_{k}, b_{k}$ with $b_{0}=E_{n}$ are given in Table 1. Fig. 7 shows the approximation error $\rho_{4}(t)-\sqrt{t}$ and the equioscillation property. Note that the asymptotic formula for $E_{n}$ is known [27], i.e., we have

$$
E_{n} \approx 8 e^{-\pi \sqrt{2 n}} \text { for } n \rightarrow \infty
$$

\footnotetext{
${ }^{5}$ Correctly speaking $a_{k, n}$ and $b_{k, n}$, but for ease of notation we omitted the explicit dependence on $n$.
} 
Formula (14) implies

$$
0 \leq \rho_{n}\left(x^{2}\right)+E_{n}-|x| \leq 2 E_{n} \quad \text { for } \quad-1 \leq x \leq 1
$$

or

$$
0 \leq \frac{\rho_{n}\left(x^{2}\right)+x+E_{n}}{2}-\max (0, x) \leq E_{n} \quad \text { for } \quad-1 \leq x \leq 1 .
$$

For $a=b=1$, the best rational function $f(x)$ satisfying (11) is therefore $f(x)=\frac{1}{2}\left(\rho_{n}\left(x^{2}\right)+x+E_{n}\right)$ with $\alpha=E_{n}$. It should be noted that $f(x)$ has numerator degree $2 n+1$ and denominator degree $2 n$. The case of the general interval $[-a, b]$ instead of $[-1,1]$ is treated by the following

Theorem 6.1. Let $a, b>0$ and $f(x)$ a real-rational function such that

$$
0 \leq f(x)-\max (x, 0) \leq \alpha \quad \text { for } \quad-1 \leq x \leq 1
$$

Then the real-rational function

$$
f_{a, b}(x)=\left[\frac{x(b-a)+2 a b}{b+a}\right] f\left(\frac{x(b+a)}{x(b-a)+2 a b}\right)
$$

is such that

$$
0 \leq f_{a, b}(x)-\max (x, 0) \leq \alpha \max (a, b) \quad \text { for } \quad-a \leq x \leq b .
$$

Proof. The bilinear transformation $g(x)=x(b+a) /(x(b-a)+2 a b)$ maps the intervals $[-a, 0]$ and $[0, b]$ onto the intervals $[-1,0]$ and $[0,1]$, respectively. Moreover, the linear function $x(b-a)+2 a b$ is positive over $[-a, b]$ since it is positive at the endpoints. Hence

$$
0 \leq f(g(x))-\max (g(x), 0) \leq \alpha \quad \text { for } \quad-a \leq x \leq b
$$

implying

$$
0 \leq\left[\frac{x(b-a)+2 a b}{b+a}\right] f(g(x))-\max (x, 0) \leq \alpha \frac{x(b-a)+2 a b}{a+b} \text { for } \quad-a \leq x \leq b
$$

which completes the proof. Note that, if the denominator degree of $f(x)$ is $m$ and the numerator degree is $m+1$, then the same holds for $f_{a, b}(x)$.

In light of formula (16), we take $f(x)=\frac{1}{2}\left(\rho_{n}\left(x^{2}\right)+x+E_{n}\right)$ and $\alpha=E_{n}$. The function $f_{a, b}(x)$ can be conveniently written as

where

$$
f_{a, b}(x)=(\tau x+\kappa) f\left(\frac{x}{\tau x+\kappa}\right)
$$

$$
\tau=\frac{\delta_{+}(H)-\left|\delta_{-}(H)\right|}{\delta_{+}(H)+\left|\delta_{-}(H)\right|}, \quad \kappa=2 \frac{\delta_{+}(H)\left|\delta_{-}(H)\right|}{\delta_{+}(H)+\left|\delta_{-}(H)\right|} .
$$

For the function $f^{\ell}(x)=1 /\left(x^{2}+\ell\right)$, the partial fraction expansion of $f_{a, b}^{\ell}(x)$ is given by

$$
f_{a, b}^{\ell}(x)=\frac{(\tau x+\kappa)^{3}}{x^{2}+\ell(\tau x+\kappa)^{2}}=x \frac{\tau^{3}}{1+\tau^{2} \ell}+\kappa \frac{\tau^{2}\left(3+\tau^{2} \ell\right)}{\left(1+\tau^{2} \ell\right)^{2}}+\Re e\left\{\frac{\eta(\tau, \kappa, \ell)}{x-\xi(\tau, \kappa, \ell)}\right\}
$$

where

$$
\xi(\tau, \kappa, \ell)=\frac{\kappa \sqrt{\ell}}{i-\tau \sqrt{\ell}}, \quad \eta(\tau, \kappa, \ell)=\frac{\xi(\tau, \kappa, \ell)^{3}}{\kappa \ell^{2}} .
$$

Hence for the function $f(x)=\frac{1}{2}\left(\rho_{n}\left(x^{2}\right)+x+E_{n}\right)$, the transformed function $f_{a, b}(x)$ can be written as

$$
f_{a, b}(x)=\frac{1}{2}\left[x+\left(E_{n}+a_{0}\right)(\tau x+\kappa)-\sum_{k=1}^{n} a_{k} f_{a, b}^{b_{k}}(x)\right] .
$$


The partial fraction expansion of (17) is the key of Algorithm 3, since we ultimately have to calculate $f_{a, b}(Z(s))$, where $Z(s)=H(s)+H(-s)^{T}$. The linear terms of (17) all add up to the compound linear term

$$
f_{a, b}^{\text {linear }}(x)=\frac{1}{2}\left[x+\left(E_{n}+a_{0}\right)(\tau x+\kappa)-\sum_{k=1}^{n} a_{k}\left\{x \frac{\tau^{3}}{1+\tau^{2} b_{k}}+\kappa \frac{\tau^{2}\left(3+\tau^{2} b_{k}\right)}{\left(1+\tau^{2} b_{k}\right)^{2}}\right\}\right]
$$

leading to a linear term $f_{a, b}^{\text {linear }}(Z(s))=k_{1} Z(s)+k_{2} I_{p}$. The remaining terms, found by evaluating

$$
\Re e\left\{\eta\left(\tau, \kappa, b_{k}\right)\left(Z(s)-\xi\left(\tau, \kappa, b_{k}\right) I_{p}\right)^{-1}\right\} .
$$

are obtained by the state space technique described in the Appendix. Finally, the stable anti-stable decomposition of $f_{a, b}(Z(s))$ is performed in order to obtain the passivated transfer function $G(s)$. Note that for Algorithm 3 the number of poles of the passivated transfer function is $N_{G}=N(2 n+1)$. Algorithm 3 can be summarized in the following steps :

1. Calculate $\delta_{-}(H)$. If $\delta_{-}(H) \geq 0$ stop, else calculate $\delta_{+}(H)$.

2. If $\delta_{+}(H) \leq 0$ stop, else put $a=\left|\delta_{-}(H)\right|$ and $b=\delta_{+}(H)$.

3. Put $Z(s)=H(s)+H(-s)^{T}$, select $n \geq 1$ and determine the coefficients $a_{k}, b_{k}$.

4. Calculate $f_{a, b}(Z(s))$ by means of the formula (17).

5. Decompose $f_{a, b}(Z(s))$ as $f_{a, b}(Z(s))=Y_{2}^{s t a b}(s)+Y_{2}^{s t a b}(-s)^{T}$.

6. The passivated transfer function is $G(s)=Y_{2}^{s t a b}(s)+\frac{1}{2}\left(D-D^{T}\right)$.

Remark 6.1. As a last remark, it is important for the interested reader to know in which case each one of the algorithms should be used. Note that Algorithms 1,2 and Algorithm 3 apply in different cases, i.e., Algorithms 1,2 apply when we only know $\delta_{-}(H)$, whereas Algorithm 3 applies when both $\delta_{-}(H)$ and $\delta_{+}(H)$ are known. Moreover, since Algorithm 1 presents minimal realization problems, Algorithm 2 should be preferred. Next, the approximation bound for Algorithms 1,2 is $O(1 / n)$ (see Corollary 4.1), whereas the approximation bound for Algorithm 3 is $E_{n}$, which is exponential (see equation (15) and Theorem 6.1). As such, Algorithm 3 is theoretically better than Algorithm 2, but Algorithm 2 has the advantage that its coefficients are analytically known for all n, whereas the coefficients for Algorithm 3 have to be numerically calculated for all $n$. Another advantage of Algorithm 2 over Algorithm 3, is that it still offers a reasonable approximation bound when $\delta_{+}(H)$ is very large, which may happen in practice. Regarding the computational complexity of Algorithms 2 and 3, we have that, since the only operations are adding and inverting LTI systems, which are mainly matrix stacking problems, and the stable, anti-stable decomposition, which has an $O\left(N^{3}\right)$ complexity [11], we can conclude that the overall numerical complexity is $O\left(N_{G}^{3}\right)$, where $N_{G}$ is the number of poles of the passivated system.

\subsection{Numerical Examples}

6.1.1. First example

As our first example we again take the SISO Hurwitz stable minimum phase non-passive transfer function (9), but here we use Algorithm 3 with $n=4$ and the coefficients of Table 1. The passivated approximation $G(s)$ has a realization with $45=5 \times(2 n+1)$ poles. The real and imaginary parts of the original transfer function $H(s)$ vs. the passivated transfer function $G(s)$ are shown in Figures 8 and 9. For comparison purposes, we also added the plots resulting from the passivity compensation algorithm of [7]. It is seen by comparing with Figures 3 and 4 that the approximation is better, while requiring 5 poles less.

\subsubsection{Second example}

For the second example we again take the MIMO Hurwitz stable non-passive transfer function (10), but here we use Algorithm 3 with $n=4$ and the coefficients of Table 1. The passivated approximation $G(s)$ has a realization with $54=6 \times(2 n+1)$ poles. Fig. 10 plots the values of $\lambda_{\min }\left(G(i \omega)+G(i \omega)^{H}\right)$ vs. $\lambda_{\min }\left(H(i \omega)+H(i \omega)^{H}\right)$. To show the nearness of the original and passivated transfer functions $H(s)$ and $G(s)$, we plot the relative error $\|G(i \omega)-H(i \omega)\|_{2} /\|H(i \omega)\|_{2}$ in Fig. 11. For comparison purposes, we also added the plots resulting from the passivity compensation algorithm of [7]. It is seen by comparing with Figures 5 and 6 that the approximation is more or less similar, but requires 6 poles more. 


\subsubsection{Third example}

As a last example we take a non-passive random state space model with 2 ports and 200 poles, and use Algorithm 3 with $n=4$ and the coefficients of Table 1. The passivated approximation $G(s)$ has a realization with $1800=200 \times(2 n+1)$ poles. Fig. 12 plots the values of $\lambda_{\min }\left(G(i \omega)+G(i \omega)^{H}\right)$ vs. $\lambda_{\min }\left(H(i \omega)+H(i \omega)^{H}\right)$.

\section{CONCLUSION}

We have presented a new global passification approach towards finding a passive real-rational transfer function $G(s)$ that is an arbitrarily close approximation of the passive transfer function nearest to the nonpassive transfer function $H(s)$. It is shown that the key point in constructing the nearest passivated transfer function $G(s)$, is to find a good rational approximation to the well-known ramp function over an interval defined by the minimum and maximum dissipation of the given non-passive transfer function $H(s)$. It is also shown that in the Chebyshev or minimax sense this requires finding a rational Chebyshev approximation of the square root function over the unit interval. The proposed algorithms rely strongly on the stable anti-stable decomposition of a given transfer function. Six pertinent examples, both SISO and MIMO, are given to show the accuracy and relevance of the proposed algorithms. Future avenues of research could be a generalization of the present results to descriptor systems and also, hopefully, to treat the related nonexpansivity approximation problem in the bounded-real case.

\section{Acknowledgement}

The author wants to thank Dr. Dirk Deschrijver for providing and sharing useful passivity enforcement and compensation code.

\section{APPENDIX}

Suppose we have the real-rational transfer function $H(s)=C\left(s I_{n}-A\right)^{-1} B+D$, and we need to find a state space realization of the transfer function

$$
\Re e \eta\left(H(s)-\xi I_{p}\right)^{-1}=\frac{1}{2} \eta\left(H(s)-\xi I_{p}\right)^{-1}+\frac{1}{2} \bar{\eta}\left(H(s)-\bar{\xi} I_{p}\right)^{-1}
$$

where $\eta$ and $\xi$ are complex numbers. Suppose also that $D-\xi I_{p}$ is invertible. Putting $D_{\xi}=D-\xi I_{p}$, we have [15] that the complex state space transfer function $\eta\left(H(s)-\xi I_{p}\right)^{-1}$ is given by $\tilde{C}\left(s I_{n}-\tilde{A}\right)^{-1} \tilde{B}+\tilde{D}$ where

$$
\tilde{A}=A-B D_{\xi}^{-1} C, \quad \tilde{B}=\eta B D_{\xi}^{-1}, \quad \tilde{C}=-D_{\xi}^{-1} C, \quad \tilde{D}=\eta D_{\xi}^{-1} .
$$

Splitting $\tilde{H}(s)=\tilde{C}\left(s I_{n}-\tilde{A}\right)^{-1} \tilde{B}+\tilde{D}$ into real and imaginary parts gives

$$
\begin{aligned}
{\left[\begin{array}{cc}
\Re e(\tilde{H}(s)) & -\Im m(\tilde{H}(s)) \\
\Im m(\tilde{H}(s)) & \Re e(\tilde{H}(s))
\end{array}\right]=} & {\left[\begin{array}{cc}
\Re e(\tilde{C}) & -\Im m(\tilde{C}) \\
\Im m(\tilde{C}) & \Re e(\tilde{C})
\end{array}\right]\left[\begin{array}{cc}
\Re e\left(s I_{n}-\tilde{A}\right) & -\Im m\left(s I_{n}-\tilde{A}\right) \\
\Im m\left(s I_{n}-\tilde{A}\right) & \Re e\left(s I_{n}-\tilde{A}\right)
\end{array}\right]^{-1}\left[\begin{array}{cc}
\Re e(\tilde{B}) & -\Im m(\tilde{B}) \\
\Im m(\tilde{B}) & \Re e(\tilde{B})
\end{array}\right] } \\
& +\left[\begin{array}{cc}
\Re e(\tilde{D}) & -\Im m(\tilde{D}) \\
\Im m(\tilde{D}) & \Re e(\tilde{D})
\end{array}\right]
\end{aligned}
$$

whose $(1,1)$-element for real $s^{6}$ is given by

$$
\Re e(\tilde{H}(s))=[\Re e(\tilde{C})-\Im m(\tilde{C})]\left(s I_{n}-\left[\begin{array}{cc}
\Re e(\tilde{A}) & -\Im m(\tilde{A}) \\
\Im m(\tilde{A}) & \Re e(\tilde{A})
\end{array}\right]\right)^{-1}\left[\begin{array}{c}
\Re e(\tilde{B}) \\
\Im m(\tilde{B})
\end{array}\right]+\Re e(\tilde{D}) .
$$

\footnotetext{
${ }^{6}$ The Laplace variable $s$ is also known as operator variable, either the derivative operator $s=d / d t$ or $\left(\right.$ for $\left.s^{-1}\right)$ the integration operator. Hence $s$ is the symbol of a real operator, and we can take $s$ real.
} 
Hence the state space equations for the transfer function (18) are given by :

$$
\begin{aligned}
\dot{x}_{1} & =\Re e(\tilde{A}) x_{1}-\Im m(\tilde{A}) x_{2}+\Re e(\tilde{B}) u \\
\dot{x}_{2} & =\Im m(\tilde{A}) x_{1}+\Re e(\tilde{A}) x_{2}+\Im m(\tilde{B}) u \\
y & =\Re e(\tilde{C}) x_{1}-\Im m(\tilde{C}) x_{2}+\Re e(\tilde{D}) u .
\end{aligned}
$$

\section{References}

[1] B. Anderson and S. Vongpanitlerd, Network Analysis and Synthesis, NJ: Prentice-Hall, 1973.

[2] S. Grivet-Talocia, Passivity enforcement via perturbation of Hamiltonian matrices, IEEE Trans. Circuits Syst. I 51 (9) (2004) 1755-1769.

[3] B. R. Andrievskii, A. L. Fradkov, The passification method in problems of adaptive control, estimation, and synchronization, Autom. Remote Control 67 (11) (2006) 1699-1731.

[4] C. J. Damaren, H. J. Marquez, A. G. Buckley, Optimal strictly positive real approximations for stable transfer functions, IEE Control Theory and Applications 143 (6) (1996) 537-542.

[5] B. Dumitrescu, Parameterization of positive-real transfer functions with fixed poles, IEEE Trans. Circuits Syst. I 49 (4) (2002) 523-526.

[6] C. P. Coelho, J. Phillips, L. M. Silveira, A convex programming approach for generating guaranteed passive approximations to tabulated frequency-data, IEEE Trans. Computer-Aided Design 23 (2) (2004) 293-301.

[7] D. Saraswat, R. Achar, M. S. Nakhla, Global passivity enforcement algorithm for macromodels of interconnect subnetworks characterized by tabulated data, IEEE Trans. VLSI Systems 13 (7) (2006) 819-832.

[8] Y. Tanji, H. Kubota, Passive approximation of tabulated frequency data by Fourier expansion method, in: Proc. ISCAS, vol. 6, 2005, pp. 5762-5765.

[9] N. J. Higham, Matrix nearness problems and applications, in: Applications of Matrix Theory, University Press, 1989, pp. $1-27$.

[10] P. R. Halmos, Positive approximants of operators, Indiana Univ. Math. J. 21 (1971/72) 951-960.

[11] B. Kågström, P. VanDooren, A generalized state-space approach for the additive decomposition of a transfer matrix, J. Numer. Linear Algebra Appl. 1 (2) (1992) 165-181.

[12] M. G. Safonov, E. A. Jonckheere, M. Vermaj, D. J. N. Limebeer, Synthesis of positive real multivariable feedback systems, International Journal of Control 45 (3) (1987) 817-842.

[13] S. Boyd, L. El Ghaoui, E. Feron, and V. Balakrishnan, Linear Matrix Inequalities in System and Control Theory, SIAM Studies in Applied Mathematics 15, Philadelphia, PA, 1994.

[14] S. Boyd, V. Balakrishnan, P. Kabamba, A bisection method for computing the $H_{\infty}$ norm of a transfer matrix and related problems, Math. Control Signals Systems 2 (3) (1989) 207-219.

[15] Control System Toolbox User's Guide, Version 4, The MathWorks, Inc, 1998.

[16] X. Chen, J. T. Wen, Positive realness preserving model reduction with $H_{\infty}$ norm error bounds, IEEE Trans. Circuits Syst. I 42 (1) (1995) 23-29.

[17] L. Knockaert, A note on strict passivity, Systems Control Lett. 54 (9) (2005) 865-869.

[18] D. C. Sorensen, Passivity preserving model reduction via interpolation of spectral zeros, Systems Control Lett. 54 (4) (2005) 347-360.

[19] A. C. Antoulas, A new result on passivity preserving model reduction, Systems Control Lett. 54 (4) (2005) 361-374.

[20] L. Knockaert, T. Dhaene, F. Ferranti, D. DeZutter, Model order reduction with preservation of passivity, non-expansivity and Markov moments, Systems Control Lett. 60 (1) (2011) 53-61.

[21] L. Knockaert, D. DeZutter, On the complex symmetry of the Poincaré-Steklov operator, Progress in Electromagnetic Research B. 7 (2008) 145-157.

[22] D. J. Newman, Rational approximation to $|x|$, Michigan Math. J. 11 (1964) 11-14.

[23] L. Brutman, E. Passow, On rational approximation to $|x|$, Constr. Approx. 13 (1997) 381-391.

[24] R. S. Varga, A. Ruttan, A. D. Karpenter, Numerical results on the best uniform rational approximations of the function $|x|$ on the interval [-1, 1], Mat. Sb. 182 (11) (1991) 1523-1541.

[25] A. Ralston, Rational Chebyshev approximation by Remes algorithms, Numer. Math. 7 (1965) 322-330.

[26] H. P. Blatt, A. Iserles, E. B. Saff, Remarks on the behaviour of zeros of best approximating polynomials and rational functions, in: Algorithms for approximation, Inst. Math. Appl. Conf. Ser. New Ser., 10, Oxford Univ. Press, 1987, pp. $437-445$.

[27] H. R. Stahl, Best uniform rational approximation of $x^{\alpha}$ on [0,1], Acta Math. 190 (2003) 241-306. 
Table 1: Coefficients for the function $\rho_{n}(t)$ for $n=4$

\begin{tabular}{c|c|c|c|c|c}
$k$ & 0 & 1 & 2 & 3 & 4 \\
\hline$a_{k}$ & 2.6397296257 & $1.4034219887 \times 10^{-6}$ & 0.0003730797 & 0.0290141901 & 5.6266532592 \\
\hline$b_{k}$ & 0.0007365636 & 0.0000917473 & 0.0049831021 & 0.1014048457 & 2.4866930733
\end{tabular}

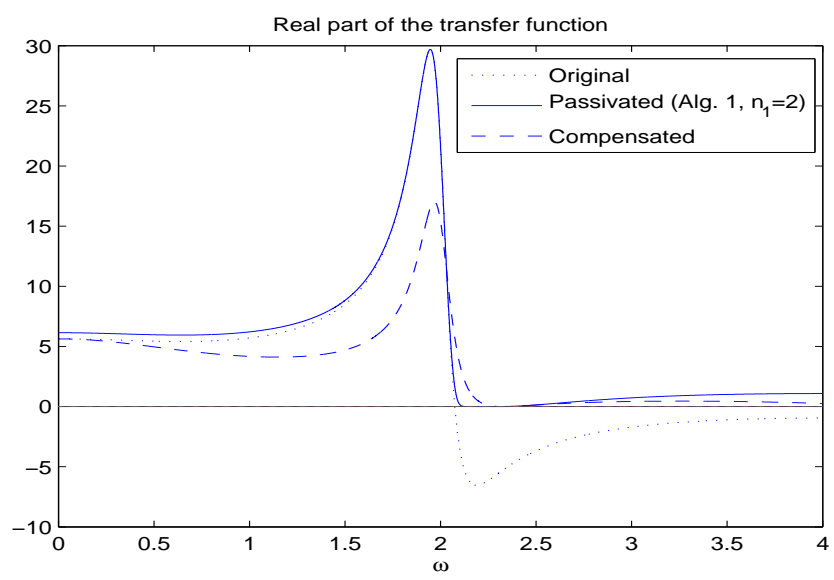

Figure 1: Real part of passivated and compensated transfer functions [7] vs. original transfer function

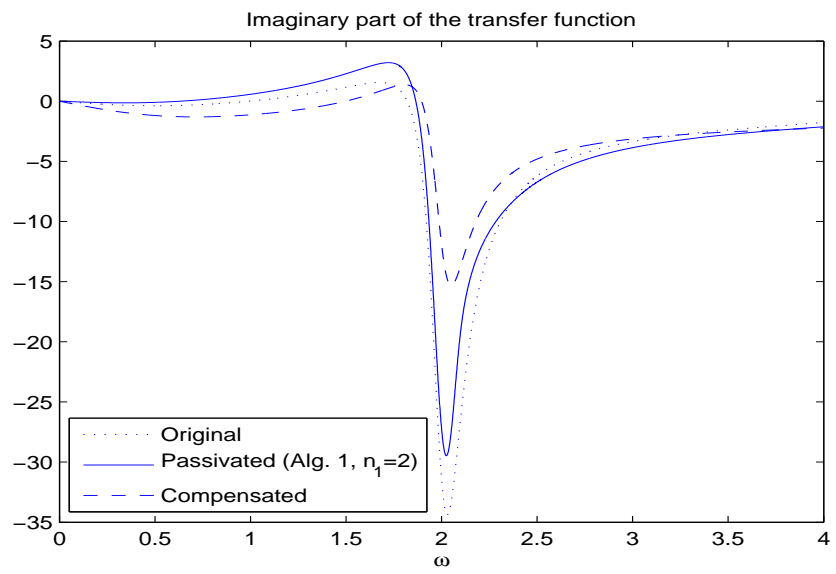

Figure 2: Imaginary part of passivated and compensated transfer functions [7] vs. original transfer function 


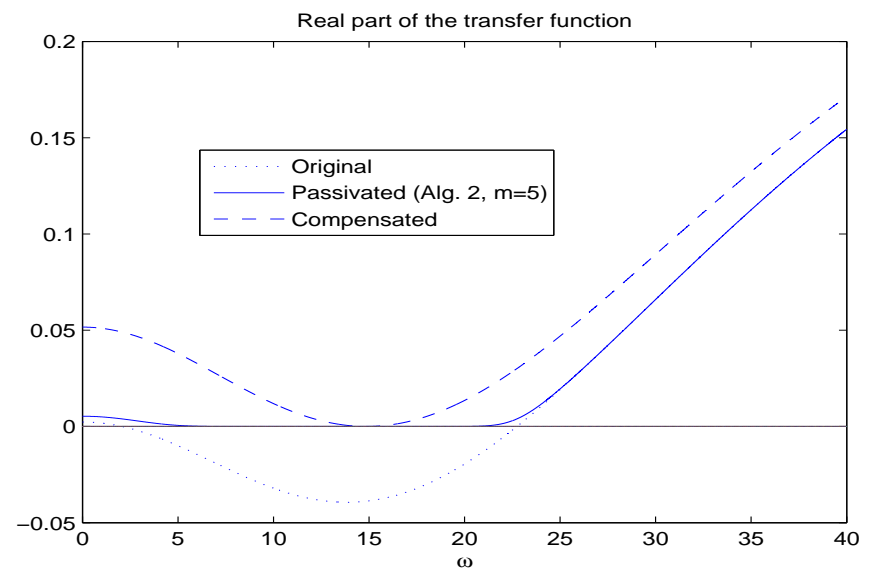

Figure 3: Real part of passivated and compensated transfer functions [7] vs. original transfer function

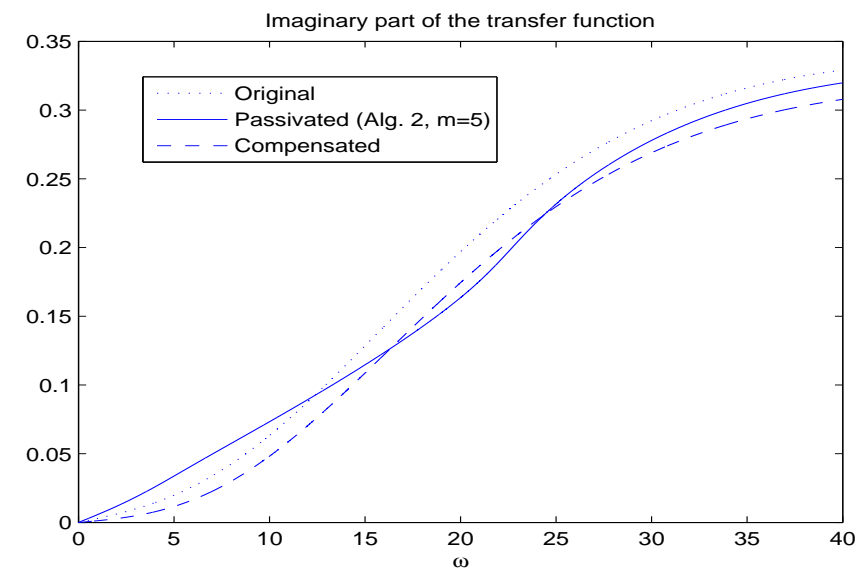

Figure 4: Imaginary part of passivated and compensated transfer functions [7] vs. original transfer function 


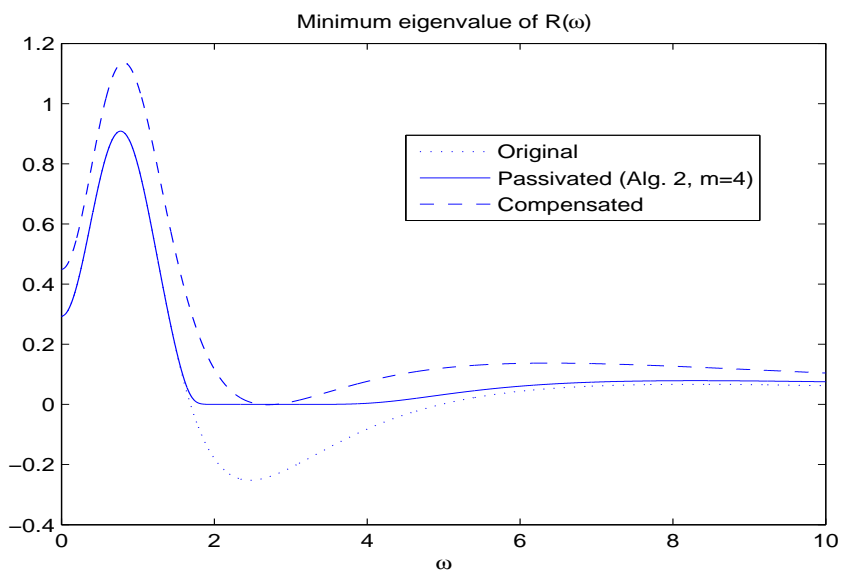

Figure 5: Minimum eigenvalue for the passivated, compensated [7] and original transfer function

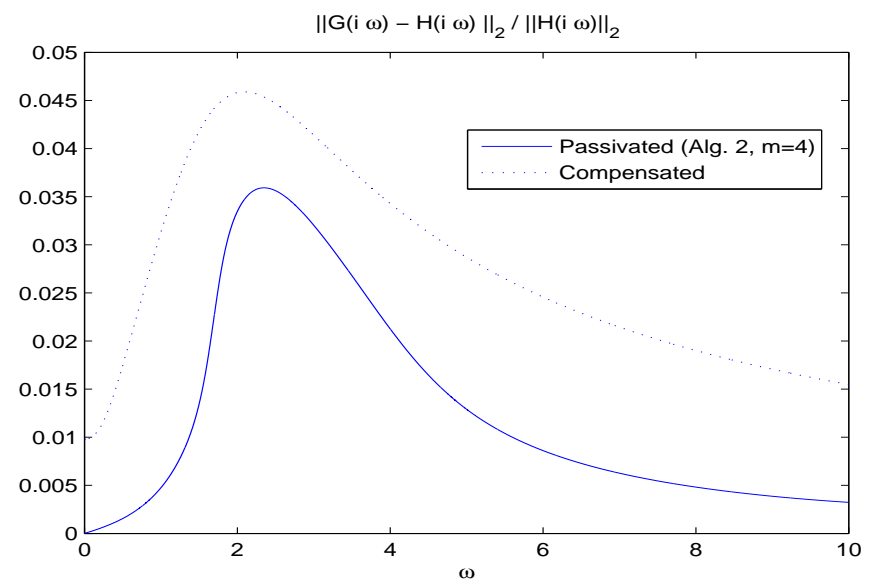

Figure 6: Relative error between passivated, compensated [7] and original transfer functions 


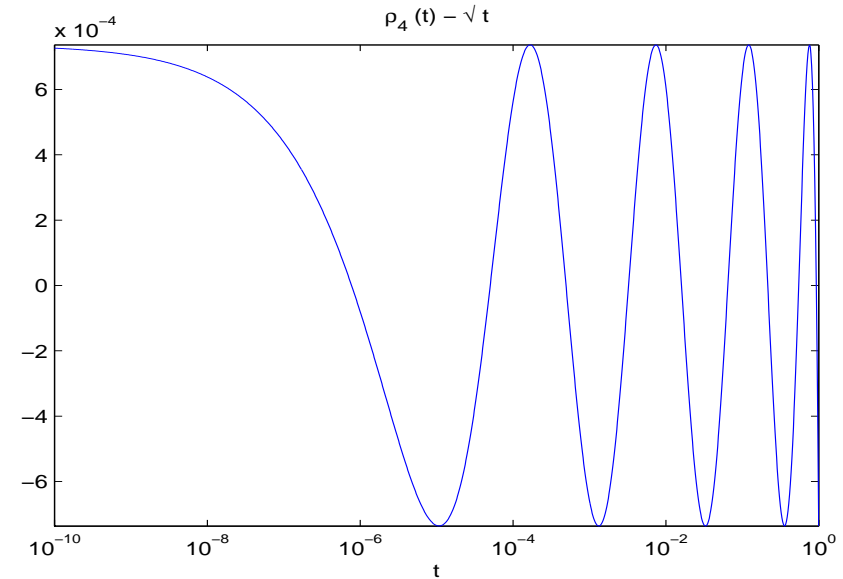

Figure 7: Minimax approximation error for $n=4$.

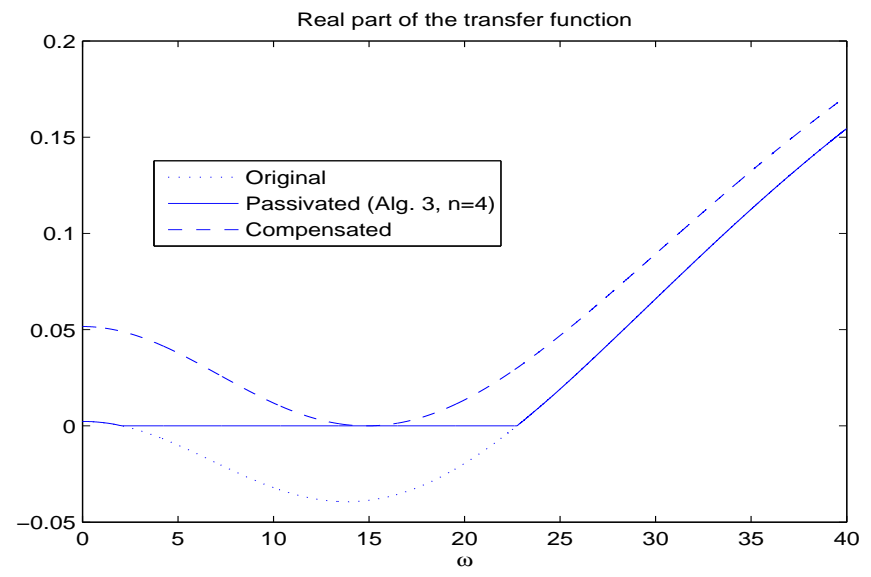

Figure 8: Real part of passivated and compensated transfer functions [7] vs. original transfer function 


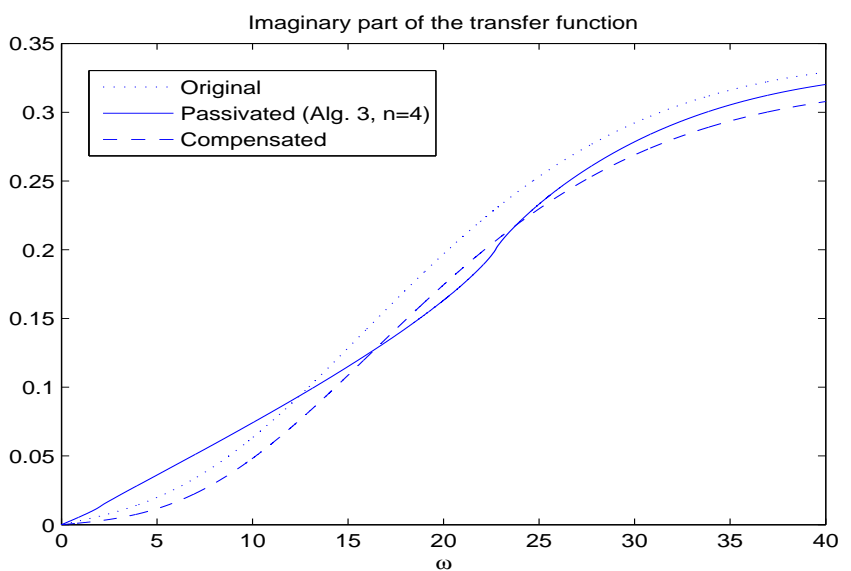

Figure 9: Imaginary part of passivated and compensated transfer functions [7] vs. original transfer function

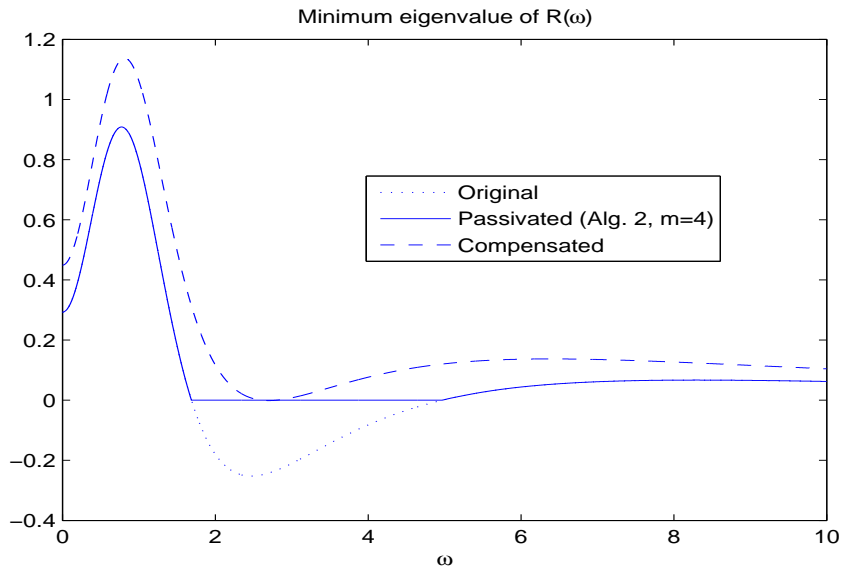

Figure 10: Minimum eigenvalue for the passivated, compensated [7] and original transfer function 


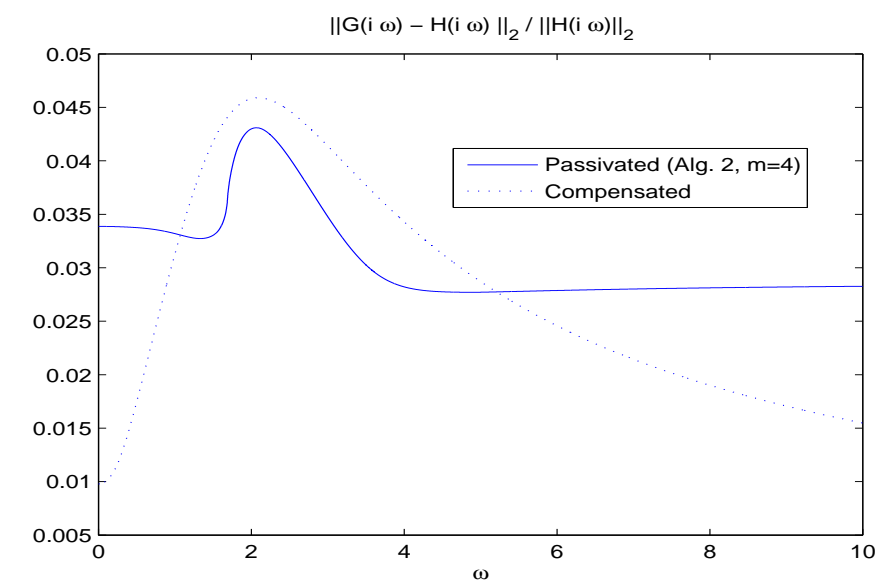

Figure 11: Relative error between passivated, compensated [7] and original transfer functions

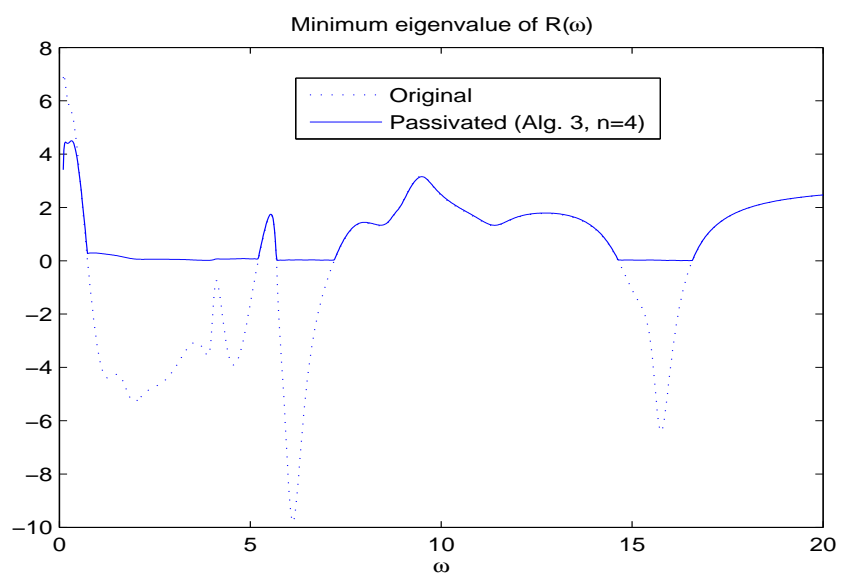

Figure 12: Minimum eigenvalue for the passivated and original transfer function 